\title{
Simulations of flowering time displacement between two cytotypes that form inviable hybrids
}

\author{
P. VAN DIJK* AND R. BIJLSMA \\ Department of Genetics, University of Groningen, PO Box 14, NL-9750 AA Haren, The Netherlands
}

\begin{abstract}
The evolution of reproductive isolation by flowering time displacement between two cytotypes that produce inviable hybrids was studied by computer simulations in an isolation-by-distance model. Flowering time distribution was stabilized by mass-action, both by the mating procedure and by pollen-limited seed-production in early or late flowering plants. Coexistence had to last long enough for flowering time divergence to evolve. This could only be achieved in a mosaic of local patches or parapatry. The model showed that flowering time displacement occurred despite the stabilizing effect of mass-action and the restricted width of the interaction zone. Initially an inverse cline for flowering time was formed but after 200 generations this cline had become monotonic. In contrast to the possible swamping effect by gene flow from outside the zone, there was a spread of genes into the allopatric ranges.
\end{abstract}

Keywords: flowering time, hybrid zone, polyploidy, reproductive character displacement, simulation.

\section{Introduction}

In the grassland perennial Plantago media both diploid and tetraploid cytotypes occur. Almost all triploid hybrids between the cytotypes are inviable because of endosperm collapse (van Dijk \& van Delden, 1990). In an area in the Pyrenees where diploids and tetraploids are sympatric, the cytotypes are almost completely separated in flowering time (van Dijk, 1991; van Dijk et al., 1992). This may explain the local coexistence of cytotypes because otherwise frequency-dependent production of inviable hybrids would lead to exclusion of the minority cytotype (Levin, 1975). How could such flowering time differences evolve? Because $P$. media is wind-pollinated, flowering time differences cannot be explained by competition for insect pollinators as has been suggested by Levin \& Anderson (1970). Because $P$. media is strictly outcrossing due to self-incompatibility, the hybridization handicap might be severe and differences in flowering time might have evolved due to reproductive interactions between cyto-

*Correspondence: Department of Plant Population Biology, Netherlands Institute for Ecological Research, PO Box 40, NL-6666 ZG, Heteren, The Netherlands. types. In a sympatric cytotype population, seed-set was negatively correlated with flowering date in diploids but positively correlated in tetraploids (van Dijk et al., 1994). This suggests selection for earlier flowering in diploids and for later flowering in tetraploids. Low seed-set was primarily due to seed abortion and endosperm collapse, suggesting that seed-set was reduced by cytotype hybridization.

A major problem in evolutionary studies is that the events often occur on a time scale beyond the observation period of the investigator. Theoretical models may then be the only possibility to gain insight into such evolutionary processes. In this article we investigate by computer simulation models whether flowering time differences could evolve as a consequence of reproductive interactions between cytotypes.

The view that natural selection may strengthen reproductive isolation between incipient species has been popularized principally by Dobzhansky (1951). In Dobzhansky's words: 'if, for example, the hybrids between species A and B are partly sterile, it is advantageous for these species to modify their breeding seasons in such a way that as few such hybrids be produced as possible'. This evolutionary process has been described in the literature as reinforcement of reproductive isolation (reinforcement for short) (Blair, 1955) 
or reproductive character displacement (Levin, 1970). Since the concept originally dates back to Wallace, Grant (1966) has suggested the use of the term Wallace effect for it. Experiments, mainly with Drosophila species, have shown that pre-zygotic isolation can indeed be strengthened by selection against hybridization (e.g. Koopman, 1950). In plants, Paterniani (1969) achieved complete flowering time separation between two corn varieties within five generations by selecting against hybrids. However, in these experiments no initial gene flow was allowed between the incipient species. Later theoretical studies have shown that strengthening of pre-zygotic isolation is unlikely if hybrids can backcross with the incipient species (e.g. Caisse \& Antonovics, 1978; Felsenstein, 1981; Butlin, 1987; Sanderson, 1989). Gene flow and recombination cause the breakdown of the associations between the genes for assortative mating and the genes that determine hybrid unfitness. Therefore, Butlin (1987, 1989) has suggested a distinction between reinforcement of reproductive isolation, in which intial gene flow was possible, and reproductive character displacement, in which gene flow was blocked intially by post-zygotic barriers. After reviewing cases of proposed reinforcement in nature, Butlin $(1987,1989)$ concluded that, in agreement with theoretical considerations, well substantiated examples of reinforcement are lacking.

Although reproductive character displacement is not hindered by recombination between the incipient species, two main theoretical problems exist. Firstly, hybrid inviability or sterility itself causes instability of sympatric populations (e.g. Crosby, 1970; Levin, 1975; Spencer et al., 1986). As hybrid production is frequency-dependent, the minority type will be rapidly eliminated, possibly before any significant character displacement has evolved. In experiments such as those of Koopman (1950) and Paterniani (1969) the ratios of the types were kept artificially constant. Two abutting parapatric cytotype populations will form a stable situation because across the border each cytotype experiences a minority disadvantage (Crosby, 1970; Butlin, 1987). However, in this case the interaction will be restricted to a narrow contact zone. Character displacement may therefore also be restricted to the contact zone or possibly even here it may be swamped by the influx of genes from the allopatric areas (Barton \& Hewitt, 1985; Sanderson, 1989).

A second major problem in the evolution of flowering time displacement is that flowering time itself is likely to be under stabilizing selection and this will operate against displacement. Moore $(1979,1982)$ has argued that the less frequent genotypes are less likely to find mates in the population. For example, in plants seed-set may be limited early and late in the flowering season due to low pollen density. In so-called 'mass- action' models, polymorphism at assortative mating loci is lost (Moore, 1979), owing to frequency-dependent mating probabilities. In contrast, in so-called 'constant fraction' models a fraction of the population mates assortatively whereas the rest mates at random (Caisse \& Antonovics, 1978; Felsenstein, 1981). In these constant fraction models, there is no disadvantage for the minority mating type. Moore (1979) therefore considered these models as less realistic.

Flowering time is a special kind of mate recognition system because it affects both male and female components (Butlin, 1989). Both single and multilocus models have been used for theoretical studies of flowering time evolution. Caisse \& Antonovics (1978) used a single locus constant fraction model whereas Moore (1982) used a single locus mass-action model for flowering time. These were general models without a population structure. Multilocus flowering time simulation models have been used by Crosby (1970) and Stam (1982), taking both flowering onset and duration into account. These models also included restricted gene flow and population structure. However, although they were not constant-fraction models, neither incorporated mass-action on flowering time.

Of these four studies only in those of Caisse \& Antonovics (1978) and Crosby (1970) was selection for reproductive isolation by flowering time studied. Allowing initial gene flow, these models can be classified as reinforcement models. Moore (1982) was interested in the colonization of early flowering plants along a selection gradient whereas Stam (1982) studied how the environment could trigger flowering time differentiation. Caisse \& Antonovics (1978) showed that the conditions for the evolution of flowering time isolation along a selective cline were quite restrictive. Crosby's simulations suggested that reinforcement by flowering time could occur, although this was a very slow and weak process. As Crosby only completed a single simulation run, it is not clear to what extent his model was affected by stochastic events. Crosby's simulation results are considered to exemplify that reinforcement may be possible (Butlin, 1987, 1989). However, when we used similar mating schemes to those of Crosby and Stam, the flowering time distributions had the tendency to segregate by themselves, in the absence of interactions with other cytotypes or with the environment. The inherent instability of the flowering time distributions may have affected the flowering time divergence in their models.

We are not aware of the existence of multilocus models of flowering time displacement incorporating mass-action. Here we describe stochastic multilocus models of flowering time in populations with restricted pollen and seed flow, both with and without massaction. Before investigating interactions between cyto- 
types, the stability of flowering time distributions in the absence of cytotype interactions was studied.

\section{The model}

We used a stochastic isolation-by-distance model, similar to those of Rohlf \& Schnell (1971), Turner et al. (1982) and Sokal \& Wartenberg (1983). A simplified flow diagram is given in Fig. 1.

The plants were considered to be annual hermaphrodites, incapable of self-fertilizing. Generations were discrete, non-overlapping. Pollination resembled wind-pollination. Plants belonged either to cytotype A

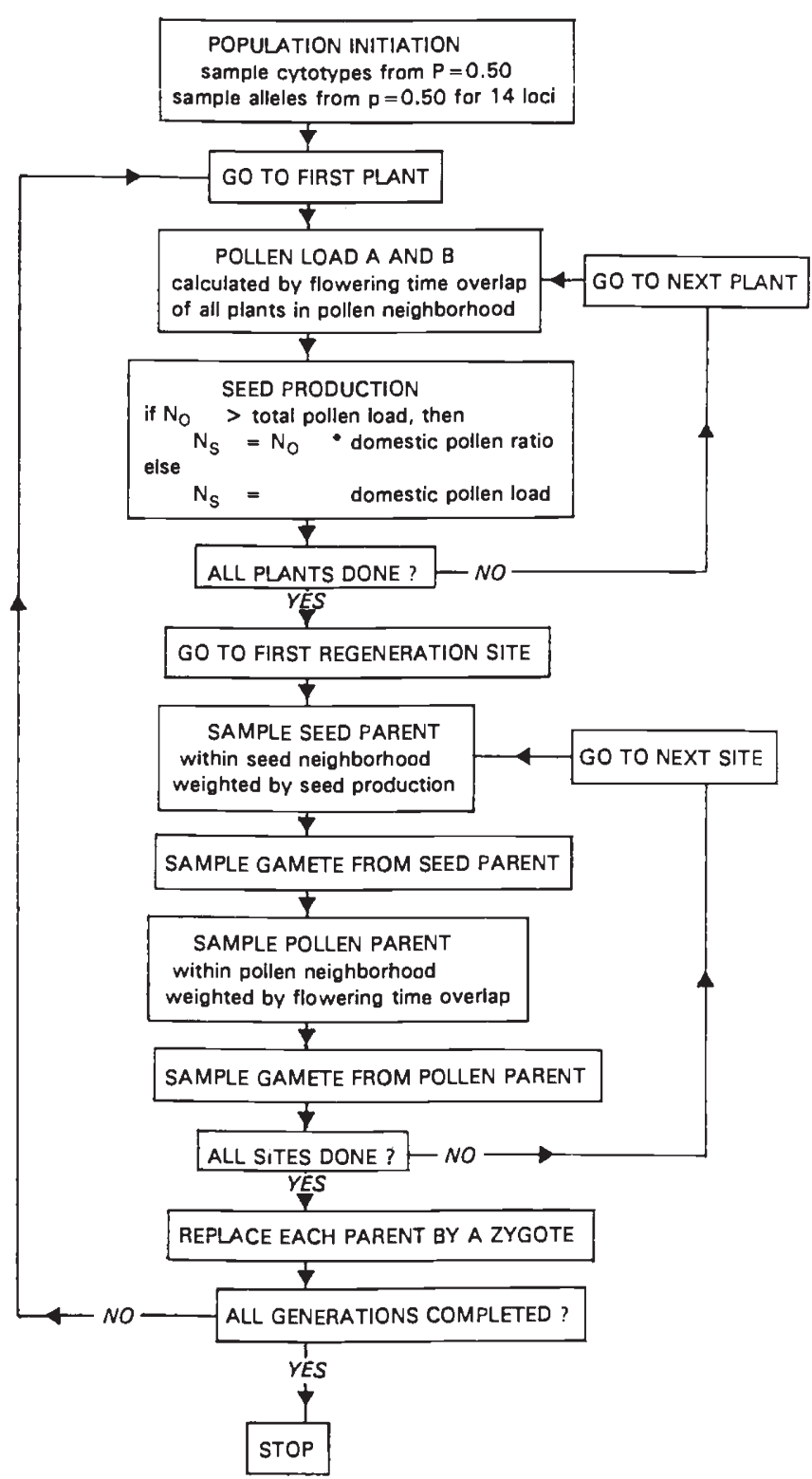

Fig. 1 Simplified flow diagram of the two-cytotype simulation models with overlap weighted sampling. or cytotype B; both cytotypes were diploid. Hybrids between the cytotypes were inviable.

Unless otherwise indicated the initial frequencies of $A$ and $B$ were equal $(0.50)$. Two different spatial starting patterns were used for the two-cytotype runs: parapatric and sympatric. In the sympatric starting pattern, cytotypes were randomly distributed over a lattice of $60 \times 50$. All sites were occupied; the total density therefore was constant. In the sympatric case the lattice was constructed as a complete torus, therefore there were no edge effects. The parapatric model consisted of two adjacent $50 \times 30$ lattices of different cytotype (see Fig. 8). In the parapatric model the upper and lower edges were connected by a torus construction. However, the left and right edges were not connected as this would have created two contact zones, which would have hindered the analysis of the processes. Plants near the left and right edges had fewer neighbours and received less pollen. In pollen limited simulations these plants produced less seeds than plants further away from these edges.

Each generation there were four stochastic events: (i) the sampling of a female parent (weighted by its seed production); (ii) the sampling of a male parent; (iii) the sampling of a female gamete and (iv) the sampling of a male gamete. These samplings were carried out by use of the pseudo-random number generator of Turbo Pascal 3.0 (Borland International Inc.).

Individual flowering onset was determined by 14 additive, unlinked loci each with two alleles $(0,1)$. Three loci had a major effect on flowering onset (major genes: 4 flowering time units delay per 1 -allele), four loci had a intermediate effect (medium genes: 2 units delay per 1-allele) while the remaining 7 loci had a minor effect (minor genes: 1 unit delay per 1-allele). Consequently flowering onset could vary between 'day' 0 to 'day' $54(=2 \times(3 \times 4+4 \times 2+7 \times 1))$. Although initial differences in flowering onset could be generated by stochastic variation, flowering onset in all plants of cytotype A was delayed 1 'day', to increase the rate of divergence. Initially alleles were sampled randomly from an allele frequency distribution of 0.50 . Loci were stored as bits and manipulated by the methods described in Fraser \& Burnell (1970). Flowering duration was the same for all individuals but could be varied between runs $(8,16$ or 32 'days'). Unless otherwise indicated flowering duration of all individuals was 16 'days'.

Both pollen and seed flow were restricted to neighbourhoods, within which mating was random with respect to distance. The neighbourhoods consisted of a sub-lattice centred on the individual to be pollinated or replaced, respectively. Individual seed production was a function of flowering synchrony within the pollen 
neighbourhood. In contrast to the models of Crosby (1970) and Stam (1982), seed production was not (necessarily) equal for all plants. Therefore the mating procedure was run first to determine seed production per plant and second to choose a male partner of a female parent. The probability that offspring of a certain plant gained a regeneration site was equal to its proportion of the total seed load at that site.

For all plants the individual flowering period was calculated from its flowering onset genotype and the general flowering duration, which was the same for each plant in a simulation run. For each plant the flowering time overlap with every potential mate in the neighbourhood was computed. The overlap determined the number of pollen grains donated by that mate (maximum 64 pollen grains with exactly synchronous flowering). Pollen thus was uniformly distributed through the plant's neighbourhood and uniformly throughout the plant's flowering period. A separate fertilizing pool of $\mathrm{A}$ and of $\mathrm{B}$ pollen was determined. When the total pollen load within a pollen neighbourhood was larger than the number of ovules to be fertilized on the central individual, seed production was set equal to the proportion of con-cytotypic pollen times the ovule number. Otherwise seed production was equal to the con-cytotypic pollen load. The number of ovules per plant could be varied between runs (Table 1). Increasing the numbers of ovules per plant caused a mass-effect because seed-set became pollen-limited in early and late flowering plants. Neigh- bourhood sizes of the different runs are given in Table 2. These sizes were chosen because of the gene flow distances measured in Plantago lanceolata (Bos et al., 1986) and the densities of flowering Plantago media in sympatric cytotype populations in the Pyrenees (Van Dijk et al., 1994).

Initially a number of runs were done with a single cytotype on a $50 \times 30$ lattice (complete torus) to analyse the evolution of flowering time in the absence of cytotypic interactions. In these single cytotype runs, two methods of choosing a partner were tested: (i) random mate choice weighted by its flowering time overlap with the seed parent (overlap weighted sampling), and (ii) randomly choosing a mate at a random moment during flowering of the seed parent (random moment sampling), comparable to the method of Stam (1982). In the two-cytotype runs only overlap weighted sampling was applied.

Most simulations were run for 200 generations; in a few runs the length was doubled. The following output data were recorded every 25 generations for both cytotypes.

1 Frequency of the cytotypes.

2 Mean population flowering onset and standard deviation.

3 Allele frequencies, $p(i)$ 's, at three major, three medium and three minor loci which were previously selected.

4 Flowering time overlap (combination of onset and duration).

Table 1 Pollen $(P)$ and ovule $\{O\}$ numbers for different $O / P$ ratios with pollen neighbourhood size of 134 self-incompatible plants $(15 \times 9-1)$

\begin{tabular}{|c|c|c|c|}
\hline $\begin{array}{l}\mathrm{O} / \mathrm{P} \text { production } \\
\text { ratio }\end{array}$ & $\begin{array}{l}\text { Pollen production } \\
\text { per plant }\end{array}$ & $\begin{array}{l}\text { Maximum pollen } \\
\text { load per plant } \dagger\end{array}$ & $\begin{array}{l}\text { Ovules } \\
\text { per plant }\end{array}$ \\
\hline 0.01 & 8576 & 8576 & 86 \\
\hline 0.20 & 8576 & 8576 & 1715 \\
\hline 0.40 & 8576 & 8576 & 3430 \\
\hline 0.50 & 8576 & 8576 & 4288 \\
\hline 1.00 & 8576 & 8576 & 8576 \\
\hline
\end{tabular}

†Assuming perfect synchronization.

Table 2 Some characteristics of the simulations

\begin{tabular}{lcccc}
\hline & & \multicolumn{2}{c}{ Neighbourhood size } & \\
\cline { 3 - 4 } & $\begin{array}{c}\text { Population } \\
\text { size }\end{array}$ & Pollen & Seed & Edges \\
\hline $\begin{array}{l}\text { One-cytotype } \\
\text { Two-cytotypes } \\
\begin{array}{l}\text { Sympatric } \\
\text { Parapatric }\end{array}\end{array}$ & $50 \times 30$ & $120(11 \times 11-1)$ & $49(7 \times 7)$ & Torus \\
\hline
\end{tabular}


5 Mean seed-set percentage per plant (since all plants produced an equal number of ovules this corresponds to seed-production and was considered as fitness).

6 In the parapatric model these data were recorded within 10 clinal blocks of $10 \times 30$ lattice points (see Fig. 8).

\section{Results and discussion}

\section{Flowering time evolution in single cytotype populations}

We first simulated flowering time evolution in a single cytotype population of 1500 plants to see how the flowering time distribution developed in the absence of cytotype interactions. The inset of Fig. 2 shows the flowering onset distribution that was initially generated. This distribution approximates to a normal distribution.

The strength of mass-action could be manipulated by changing the ovule/pollen ratios $(\mathrm{O} / \mathrm{P}$ ratios $)$. In Fig. 2 the relations between seed-set and flowering onset 'date', after the first round of fertilization, generated by $\mathrm{O} / \mathrm{P}=0.01,0.40$ and 1.00 are shown, for a pollen neighbourhood size of 120 . This can be considered as a fitness graph. For $\mathrm{O} / \mathrm{P}$ ratios of 0.40 and 1.00 , seed set is reduced in early and late flowering plants whereas for $\mathrm{O} / \mathrm{P}=0.01$, seed-set is independent of flowering onset. This can be explained as follows. Early or late flowering plants will find fewer partners that overlap in flowering time in their pollen neighbourhood than plants with intermediate flowering onset. When $\mathrm{O} / \mathrm{P}=0.01$, each plant produces 100 times more pollen grains than ovules. Within the pollen neighbourhood mating is random with respect to distance and is only determined by the flowering times. As there are 120 potential partners in the pollen neighbourhood, even single flowering plants will be likely to receive enough pollen for the fertilization of all their ovules. For $\mathrm{O} / \mathrm{P}=0.01$, then, there is pollen saturation and seed-set is pollen-unlimited (Fig. 2). For $\mathrm{O} / \mathrm{P}=0.40$, each plant produces 2.5 times as much pollen as ovules. In this case, even the modal classes of flowering onset, on average, do not receive enough pollen grains for full seed-set. Seed-set in early and late flowering plants is strongly pollen-limited. For $\mathrm{O} / \mathrm{P}=1.00$ this effect is highly enhanced.

Figure 3 shows the mean seed-set per plant at generation 0 for different ovule/pollen ratios, with a pollen neighbourhood size of 120 . For O/P values of 0.20 and higher, mean seed-set is lower than 100 per cent and selection against early or late flowering will occur.

Under pollen-saturation $(\mathrm{O} / \mathrm{P}=0.01)$, the evolution of the flowering onset distribution was markedly different between overlap weighted sampling and random moment sampling. Figure $4 \mathrm{~A}$ shows the intial flowering onset distribution whereas Fig. $4 \mathrm{~B}$ and $\mathrm{C}$ show characteristic flowering onset distributions after 200 generations under both procedures. With the overlap weighted sampling the kurtosis of the distribution increased. However, with the random moment sampling method the variation in flowering onset increased and the distribution segregated and finally became bimodal. With the latter procedure the original population diverges spontaneously into two populations that

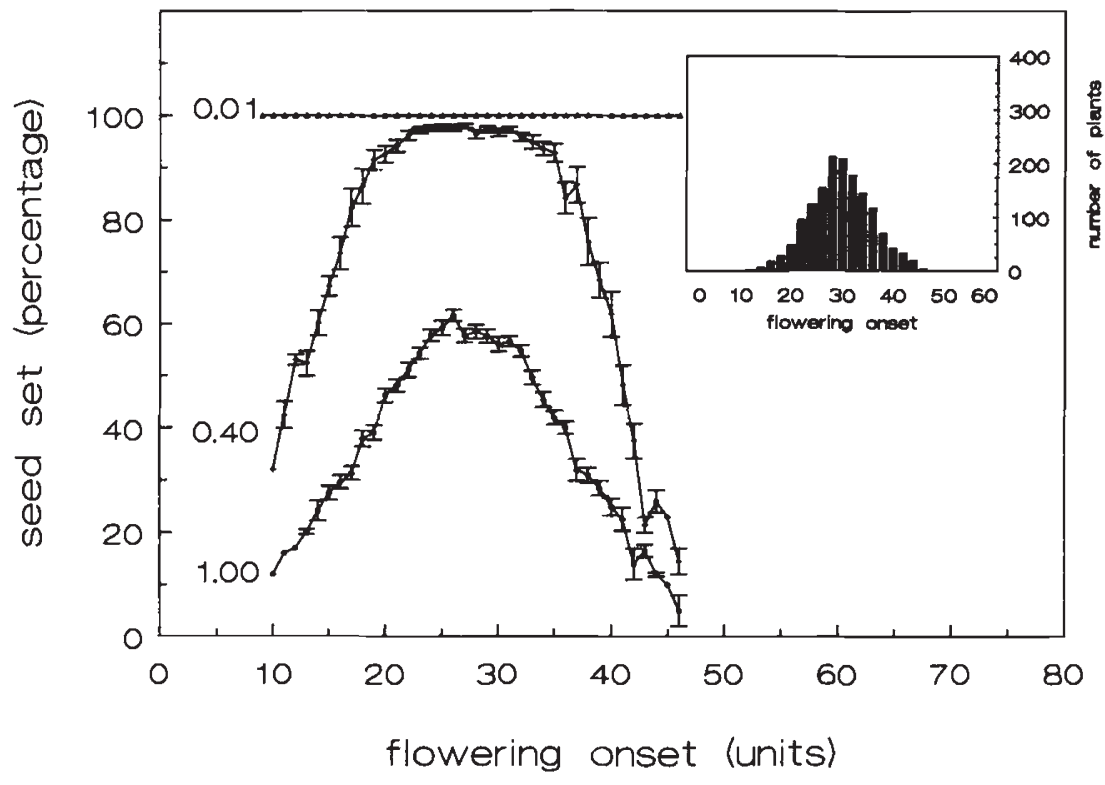

Fig. 2 Single-cytotype simulations, fitness distribution. Seed-set after the first round of fertilization in relation to the flowering onset of a plant for three different ovule/pollen ratios $(0.01,0.40$, 1.00 ), causing pollen-saturated, moderate and severe pollen-limited seed-set, respectively. Mean seed-set per flowering onset class \pm S.E. The inset shows the distribution of the flowering onset in the population. The initial allele frequencies for flowering onset $(p(i) 0$ 's $)$ were all 0.50 ; flowering duration was 16 'days' and there were 120 plants per pollination neighbourhood. 


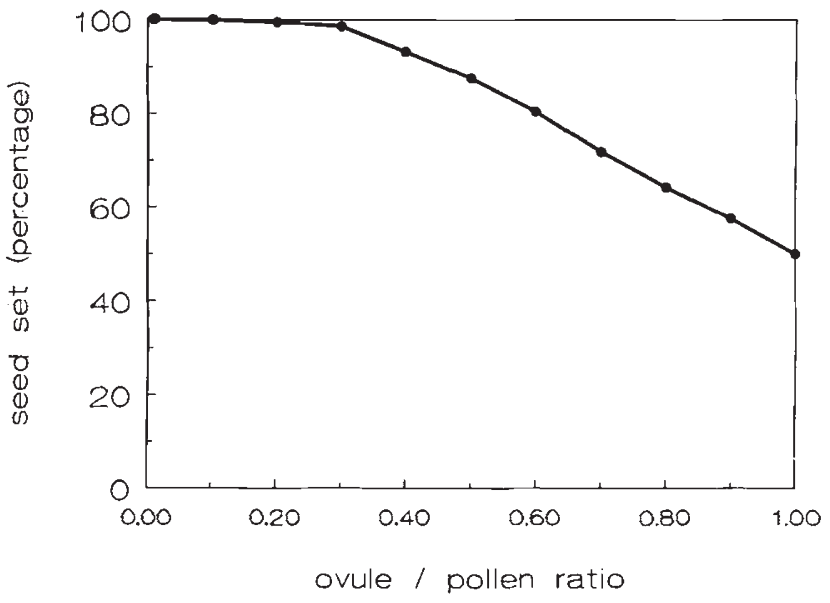

Fig. 3 Single-cytotype simulations. The mean population seed-set after the first round of fertilization for different ovule/pollen $(\mathrm{O} / \mathrm{P})$ ratios. The other parameters were similar to those described in Fig. 2.

are completely reproductively isolated by flowering period.

It is well known that positive assortative mating causes an increase in homozygosity. In a single locus model this is obvious; with perfect assortative mating of all the three genotypes, the percentage of heterozygotes is halved in each generation. In a multilocus model segregation will occur more slowly as many different genotypes will have the same phenotype but eventually the result will be multilocus homozygosity. Moreover, this segregation will also be slowed down because flowering duration allows mating of plants with different flowering onset dates.

How can the difference between the two mating schemes be explained? As intermediate flowering onset genotypes are the most frequent, these are the most likely pollen parents in either mating scheme. In the overlap weighted method the whole flowering period of the female plant is taken into consideration. This gives a stronger bias towards intermediate flowering partners than in the random moment method in which the moment chosen is independent of the number of plants flowering at that time. This is a massaction effect not caused by frequency-dependent seedset but by frequency-dependent mating. In the overlap weighted mating scheme this mass-effect overcomes the natural tendency of segregation of the flowering time distributions. In the random moment scheme, however, the mass-effect is not strong enough to prevent segregation.

Inspection of the genetic composition of the individuals clarifies what is happening under the two mating schemes. After 200 generations, under the random moment method the population consisted of multilocus


Fig. 4 Single-cytotype simulations. Typical flowering onset distributions under different mate sampling procedures at the start and after 200 generations. (A) Initial distribution (s.d. =6.87). (B) Distribution after 200 generations with random moment sampling procedure (s.d. $=18.05)$. (C) Distribution after 200 generations with the overlap weighted sampling procedure (s.d. $=3.82)$. Conditions: ovule/pollen ratio $=0.01$ (pollen saturation); $p(i) 0$ 's all 0.50 ; flowering duration: 16 'days'; pollination neighbourhood size: 120 plants; seed neighbourhood size: 49 plants.

00 or 11 homozygotes for major and medium genes $(0000000 \ldots . . . / 0000000 \ldots . .$. and $1111111 \ldots \ldots$. $1111111 \ldots . .$. ) (minor genes are indicated as .). By contrast under the overlap weighted method all plants were homozygous 00 or 11 for alternating loci (e.g. $0011101 \ldots . . . / 0011101 \ldots . . .$.$) . Minor genes were not$ yet completely fixed after 200 generations of simulation. 
The rate of segregation was shown to depend on the flowering duration. When the flowering duration was reduced from 16 to 8 'days', the segregation tendency was much stronger for the random moment method and even under the overlap weighted method there was a tendency for segregation. By contrast, a duration of 32 'days' showed little segregation after 200 generations in either mating scheme.

Figure 5 shows the change in the variation in flowering onset during the first 200 generations for random moment sampling under pollen saturation $(\mathrm{O} / \mathrm{P}=0.01)$ and for overlap weighted sampling under pollen saturation $(\mathrm{O} / \mathrm{P}=0.01)$ as well as under strong pollen limitation $(\mathrm{O} / \mathrm{P}=1.00)$. The standard deviation describes the variation of the distribution. Under pollen saturation the flowering onset variation initially increased for both mating schemes because of the development of local early and late flowering patches as the result of restricted gene flow. Local assortative mating enhanced initial chance differences and caused a patchy flowering onset landscape, as has been noticed before by Stam (1982). This pattern must not be confused with the pattern of neutral genes caused by local genetic drift in isolation-by-distance models by Rohlf and Schnell (1971) and Turner et al., (1982) as flowering time is a multilocus phenotype. Moreover, as the neighbourhood sizes in our models are rather large, local genetic drift can not have played an important role, although flowering time variation by itself will have reduced the effective neighbourhood size somewhat.

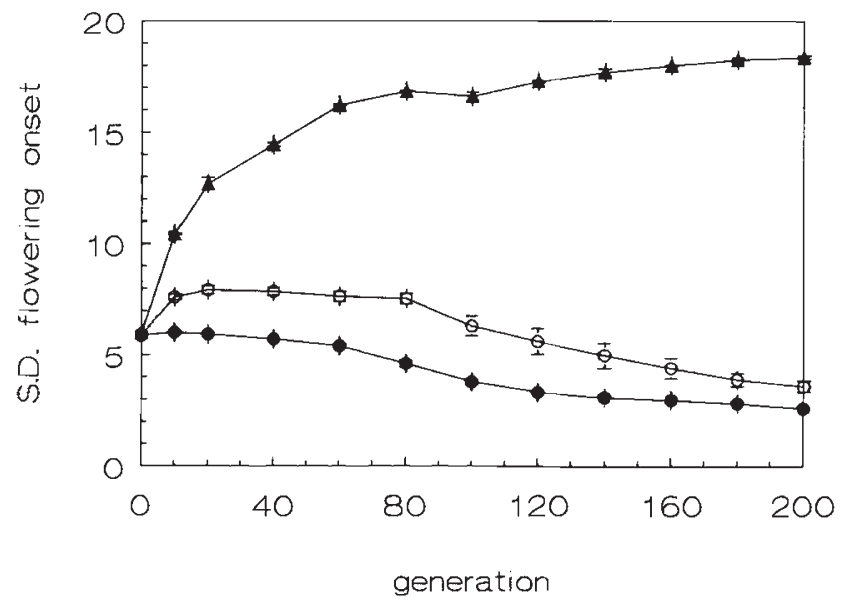

Fig. 5 Single-cytotype simulations. The change of the standard deviations of the flowering onset distributions under different mate sampling procedures during 200 generations. Random moment sampling $(\boldsymbol{\Delta})(\mathrm{O} / \mathrm{P}=0.01$; pollensaturated seed-set); overlap weighted sampling $(\mathrm{O})(\mathrm{O} / \mathrm{P}=$ 0.01 ; pollen-saturated seed-set) and overlap weighted sampling $(\bullet)(\mathrm{O} / \mathrm{P}=1.00$; severely pollen-limited seed-set $)$. Mean values of five different runs \pm S.E. per parameter set. Conditions are similar to those in Fig. 2.
While the variation in flowering onset also continued to increase after 20 generations in the random moment sampling method, it stabilized after 20 generations in the overlap weighted method (Fig. 5) and after 80 generations clearly decreased. After 200 generations the variation in flowering onset was less than the initial variation (see also Fig. 4C). Under severe pollen limitation $(\mathrm{O} / \mathrm{P}=1.00)$, the initial differences between the local flowering time patches were much smaller than under pollen saturation. Variation in flowering time decreased rather rapidly because of stabilizing selection. At the same time the mean seed-set increased from 55 per cent in generation 0 to 80 per cent in generation 200. In this case the stabilizing effect on the flowering onset distribution was caused by the effect of mass-action by biased mating and frequencydependent seed-set.

The overlap method may be less realistic than the random moment method as in this model fertilization takes place at the end of the flowering period of a plant. In reality early pollinations will pre-empt ovules for later pollinations. However, the random moment method of partner sampling cannot be used in a model with two cytotypes with inviable hybrids. Moreover, the overlap method causes a stabilizing force that prevents segregation and operates against differentiation. If despite this stabilizing force, flowering time displacement occurs, this is a robust result of the model.

Neither Crosby (1970) nor Stam (1982) reported on the stability of their flowering time distributions in the absence of the interactions they studied. Stam used a longer flowering time duration and therefore the segregation tendency in his model may not have been very strong. Crosby used a slightly different model, in which mating occurred on a weekly basis and was determined by only three onset genes and three duration genes. During his simulation flowering duration became very short, possibly by selection against sub-species hybridization. As a consequence the flowering onset distribution probably became less stable. Moreover, plants that had no overlapping flowering partner in their neighbourhood were self-fertilized, which caused segregation at all heterozygous loci. It is hard to judge how much spontaneous segregation of the flowering time distributions may have affected Stam's and Crosby's results. At least it might have facilitated flowering time differentiation. In this light it is noteworthy that in Crosby's simulations flowering time differentiation became greater than was necessary for reproductive isolation. Obviously different alleles were fixed in the two subspecies suggesting an intrinsic instability of the flowering time scheme of his model. 
Flowering time evolution in two-cytotype simulations

Sympatry. Next we investigated whether it was possible for two cytotypes to coexist long enough for flowering time displacement to evolve. In these simulations there was pollen saturation $(\mathrm{O} / \mathrm{P}=0.01)$ but seedset was reduced by cytotype hybridization. These sympatric simulations were started with a random spatial distribution of the cytotypes (see Figs 7 and 8 ).

It was found that in a sympatric starting pattern when the frequency of one cytotype was less than 0.45 , this minority cytotype was rapidly eliminated from the population. The rate of elimination was close to that in the deterministic random mating model of Levin (1975) (pollen saturation; no mass-action) (Fig. 6A). Figure 7 shows the change in spatial pattern of a typical minority cytotype elimination. Some patchiness developed due to local minority cytotype exclusion but these patches were not stable enough to persist longer than a few generations. Obviously, restricted gene-flow does not expand time of co-existence significantly.

However, when starting frequencies of the cytotypes were very close or equal to 0.50 , occasionally a pseudo-stable pattern developed (Fig. 6B). The random starting distribution caused local chance differences in cytotype frequencies. Due to the combined action of restricted gene flow and locally occurring minority cytotype elimination, discrete single cytotype patches developed. These patches became increasingly homogenous and tended to minimize the length of their contact zones, similar to the behaviour of tension zones (hybrid zones that are maintained by the balance between dispersal and selection against heterozygotes) (Barton \& Hewitt, 1985). This is to be expected, since in our simulations hybrids between cytotypes are inviable. At the same time the mean seedset of both cytotypes increased dramatically since the cytotype hybridization was reduced by distance isolation during this process.

This developing mosaic of different cytotype patches enabled co-existence of cytotypes for many generations. However, in simulations without genetic variation for flowering onset $(P(i)=1.00)$, the smaller patches disappeared in the long run and after 200 generations generally only a single cytotype persisted. Therefore this situation can be described as pseudostable. In simulations with genetic variation for flowering onset $(P(i)=0.50)$, flowering time divergence could occur before elimination of the minority type and stable coexistence was the result. The initial contraction of the cytotype patches during the first 15 generations was followed by expansion in later generations due to flowering onset differentiation. Sometimes complete flowering time separation was achieved within 30 generations. However, the development of stable sympatry was a matter of chance; often the stochastic deviations from 0.50 were too large to build up patches of approximately the same size and elimination of the minority cytotype occurred within 20-30 generations (Fig. 6B), despite the presence of genetic variation for flowering time. In six out of 10 runs one of the cytotypes was eliminated before complete flowering time isolation was achieved.

Parapatry. The process of flowering displacement can be better analysed in two abutting parapatric cytotype populations. In transects perpendicular to the contact zone, the progress of the displacement through the population can be monitored.

The initial situation is shown in Fig. 8 in which the pollen and seed neighbourhood areas are also shown on the same scale. The zone of interaction is restricted to the seed and pollen neighbourhoods along the contact zone of the two-cytotype populations and is

Fig. 6 Two-cytotype simulations.

(A) Exclusion of the minority cytotype

A, initial frequency of 0.45 , according to the random mating model of Levin (1975), without flowering onset variation $(+)$ and according to the present isolation-by-distance model with flowering onset variation and overlap sampling $(\bullet)(\mathrm{O} / \mathrm{P}=0.01$; for other conditions see the legend of Fig. 2). (B) Changes in the frequencies of cytotype A over 80 generations in four different runs when started at 0.50 , according to the isolation-by-distance model with flowering onset variation and overlap sampling $(\mathrm{O} / \mathrm{P}=0.01$; for other conditions see the legend of Fig. 2).

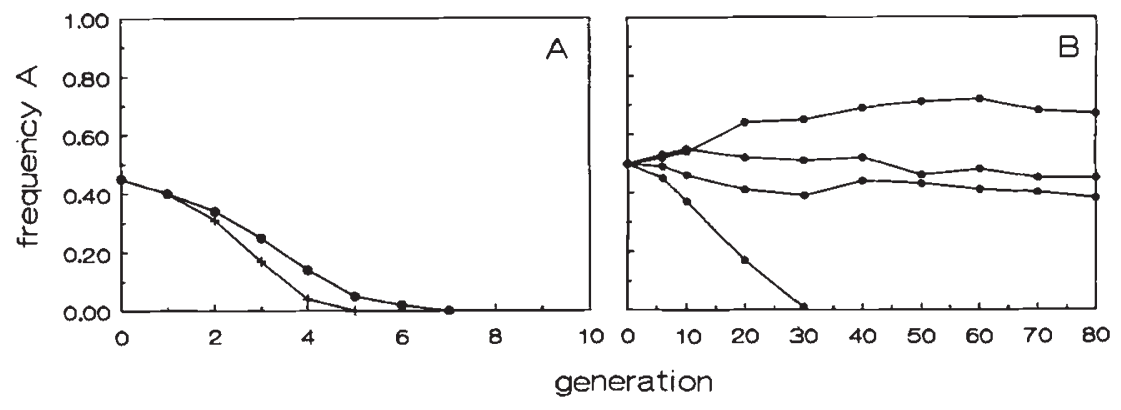






0

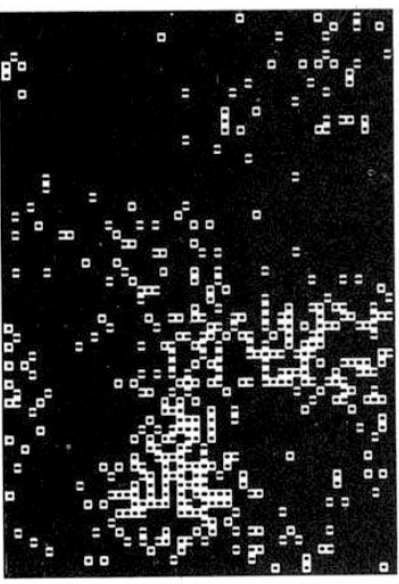

4

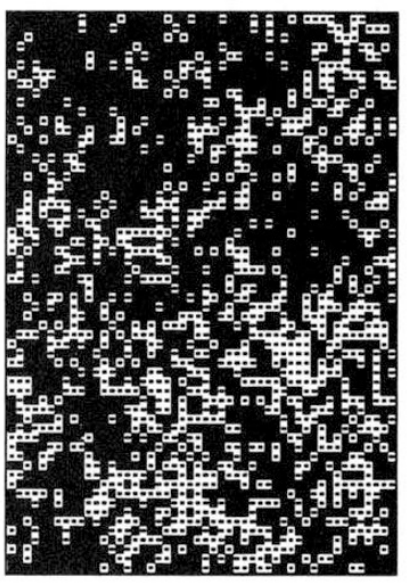

2

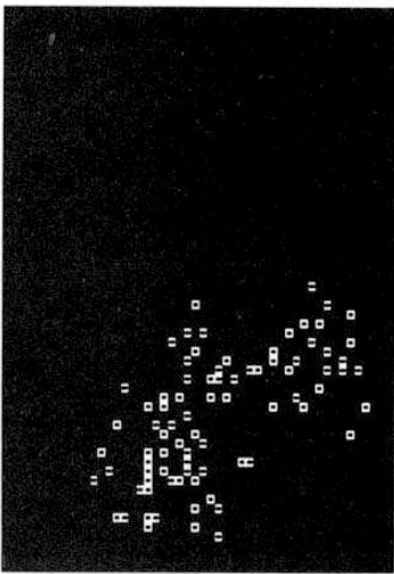

6


Fig. 7 Two-cytotype simulation: spatial distribution of cytotypes. A typical elimination pattern of a minority cytotype (initial frequency of cytotype A: 0.45 ) in sympatry after $0,2,4$ and 6 generations (dotted square: cytotype $\mathrm{A}$; black square: cytotype B; overlap weighted sampling; $\mathrm{O} / \mathrm{P}=0.01$; for other conditions see Fig. 2). The frequencies of cytotype A are displayed right at the bottom of the maps. The pollen $(\mathrm{p})$ and seed neighbourhood $(\mathrm{s})$ areas are shown on the same scale.

narrow compared with the areas without interaction. Without variation for flowering onset this parapatric distribution was shown to be stable. However, when one cytotype was given more ovules or pollen, the contact zone moved into the area of the cytotype with the fewest gametes. When a zone of 10 per cent density was modelled, this moving contact zone was trapped in the zone of low density (data not shown). This behaviour has been described for moving tension zones (Barton \& Hewitt, 1985). During our flowering time simulations, however, the cytotypes were equal except that flowering onset was delayed one 'day' in cytotype A.

First the case of pollen saturation will be considered $(\mathrm{O} / \mathrm{P}=0.01)$. Figure 8 shows the change in spatial pattern of a characteristic simulation run. The evolution of flowering time of the same run is shown in Figs 9 and 11 for the total population and for cross-sections of the population in Fig. 10. In Fig. 10 it can be seen that at generation 25 in the interaction zone blocks 5, 6 and 7) flowering time divergence has become much larger than between blocks 1 and 10. Clear inverse clines were established. On the gene level the inverse cline was much more pronounced for the major genes than for the minor genes. At the same time, intermingling was much stronger than can be explained by seed dispersal across the contact zone; obviously these intermingling cytotypes no longer hybridized because of flowering time separation. The flowering time differentiation progressed into the allopatric areas and gradually the inverse clines disappeared (Fig. 10), while the intermingling progressed more and more (Fig. 8).

Figure 11 shows the divergence of the flowering onset during the first 200 generations. The rate of flowering time divergence was slower than in the sympatric scenario, as was expected because of the smaller interaction zone. While the cytotypes diverged in flowering onset, the variation in flowering onset within the cytotypes decreased because of the massaction caused by the overlap weighted mating scheme. Spencer et al. (1986) doubted whether the process of displacement would be completed as the selective 

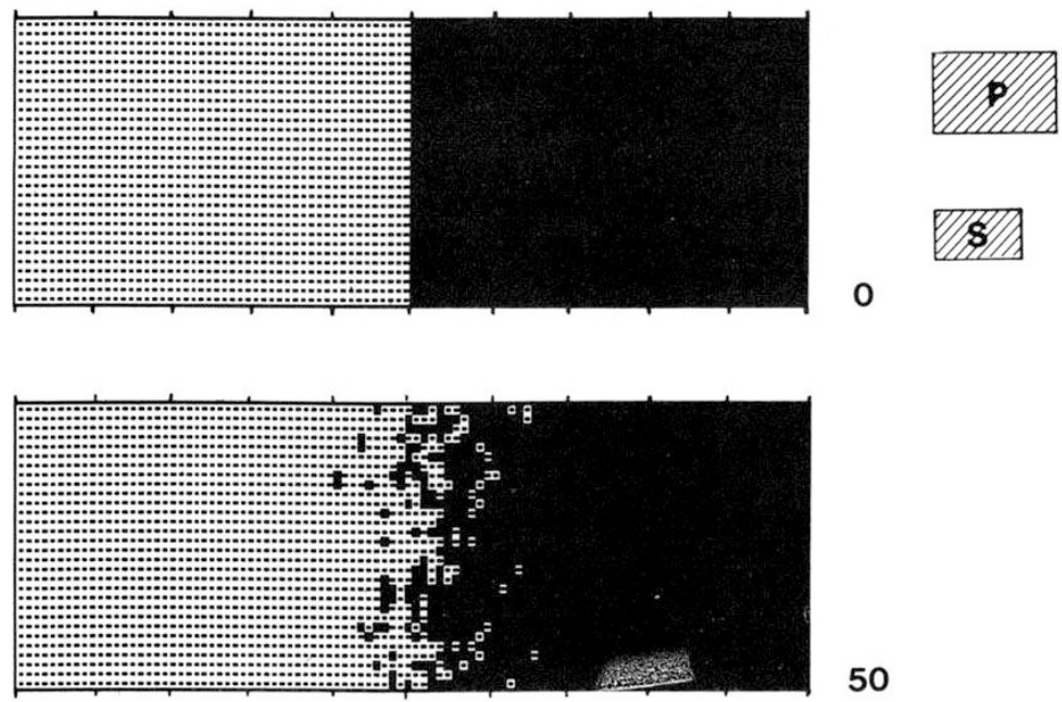

Fig. 8 Two-cytotype simulation: spatial distribution of cytotypes. The intermingling in space of two parapatric cytotype populations after $0,50,100$ and 200 generations, indicated to the right (dotted square: cytotype $\mathrm{A}$; black square: cytotype $\mathrm{B}$ ). Conditions: overlap sampling; $\mathrm{O} / \mathrm{P}=0.01$ (pollen saturation); $p(i) 0$ 's all 0.50 ; flowering duration: 16 'days', pollination neighbourhood size: 120 plants; seed neighbourhood size: 45 plants. The pollen (p) and seed neighbourhood (s) areas are shown on the same scale. The numbered blocks consist of 10 rows of 30 plants.

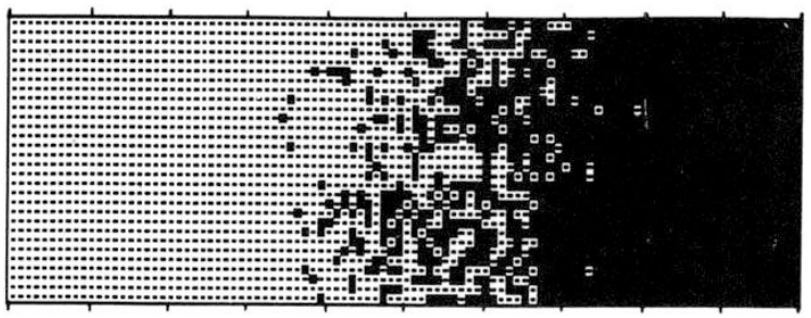

100

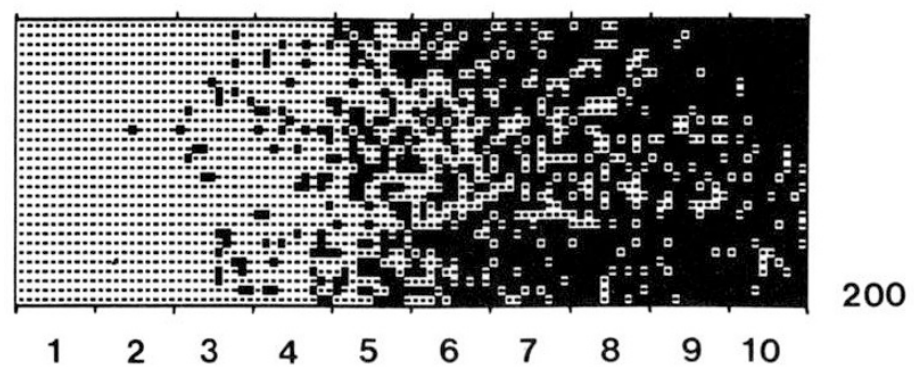

pressure would become weaker as the assortative mating characters are established. Although the rate of flowering time overlap reduction decreased, in the end, it was complete. It can be seen that there is no progress in flowering onset divergence after the average flowering time overlap had become zero (generation 160 in Fig. 11). At the same time, a considerable amount of genetic variation for flowering onset is still available as is indicated by the standard deviations in Fig. 11. Thus the model has no tendency to segregate by itself. Probably mass-action accomplished the complete separation of the flowering time distributions.

Initially cytotype A was set to flower on average 1 'day' later than cytotype B, to initiate disruptive selection. In most cases cytotype B diverged towards earlier flowering and cytotype A towards later flowering.
However, the flowering sequence that evolved was occasionally inverse to the initial sequence, indicating that genetic drift was an important factor in creating an initial difference that could be enhanced by selection.

Figure 12 shows the cross-sections of flowering onset after 200 generations under different $\mathrm{O} / \mathrm{P}$ ratios. It can be seen that the inverse cline under mass-action disappeared but that the divergence after 200 generations was less than under pollen saturation. Also the degree of intermingling was reduced under pollen limitation. With $\mathrm{O} / \mathrm{P}=0.40$ complete flowering time separation was achieved after 350-400 generations. Strong pollen-limitation, hence mass-acton (O/ $\mathrm{P}=1.00$ ), prevented flowering time displacement completely during the first 500 generations (data not shown). 



Fig. 9 Change in the flowering onset distributions in the simulation run shown in Fig. 8. Distributions after 0, 100 and 200 generations are shown together with the population means \pm s.d. (open bars: cytotype A; closed bars: cytotype B).

When the flowering time duration was doubled fiom 16 to 32 'days', no significant divergence developed under pollen saturation. Clearly flowering duration of individual plants has to be short for the development of flowering time displacement. In reality in $P$. media a low level of gene flow between diploids and tetraploids is possible because of low frequencies (about 0.1 per cent) of viable triploid and tetraploid hybrids (van Dijk, 1991). Probably gene flow is mainly unidirectional from diploids to tetraploids. A modification of the present simulation model, allowing an initial gene flow of 0.05 (gamete per generation) from A and B did not notably affect the rate of flowering time divergence. In fact this would be a case of reinforcement of reproduc- tive isolation rather than reproductive character displacement.

\section{Evolutionary perspectives of flowering time displacement}

Our simulations indicate that a polygenic assortative mating character can be displaced in populations with restricted gene flow, leading to complete pre-zygotic reproductive isolation and coexistence of cytotypes. Selection against hybridization was maximum due to hybrid inviability. This is not unrealistic as hybrids in interploidy crosses are often inviable or sterile (Woodell \& Valentine, 1961; Kay, 1969; Johnston et al., 1980; van Dijk \& van Delden, 1990).

The simulations indicate that long individual flowering periods will impede flowering time displacement. In species that have a long flowering season, flowering displacement is therefore unlikely to occur. In partially selfing species the amount of hybridization will be much reduced and consequently less flowering time displacement than in outcrossing species is to be expected.

Our models did not include environmental or developmental variation for flowering time. These factors are likely to slow down the rate of flowering time divergence between the cytotypes. Rathcke \& Lacey (1985) have argued that although flowering time can be easily altered as it is probably under simple genetic control, it is unlikely to cause effective isolation owing to phenotypic plasticity. Nevertheless, in Plantago media flowering time causes effective reproductive isolation between sympatric diploid and tetraploid cytotypes (van Dijk et al., 1994). Flowering time displacement may be constrained by other selective forces acting on flowering time (Rathcke \& Lacey 1985; Ollerton \& Lack, 1992; Fox \& Kelley, 1993). Pollinator phenology in animal pollinated species and the seasonal development of the vegetation in windpollinated plants may also restrict flowering time divergence. Flowering time divergence may also be constrained by developmental correlations between flowering time and other phenological stages in the annual cycle (e.g. the timing of leaf development in spring or the seed germination period).

The process of reproductive character displacement can be demonstrated in sympatric populations by the enhanced fitness of positive assortative mating individuals, provided that the character is heritable (van Dijk et al., 1994). However, for the demonstration that reproductive characters are displaced (the products of character displacement), geographical comparisons of the reproductive character are necessary. As a consequence of reproductive character dis- 
Fig. 10 Spread of flowering time divergence through the populations in the simulation run shown in Fig. 8. Cross-sections after $0,25,50,75,100$ and 200 generations. Mean flowering onsets per block (see Fig. 8) \pm s.d. are shown ( + : cytotype A; $\bullet$ cytotype B).
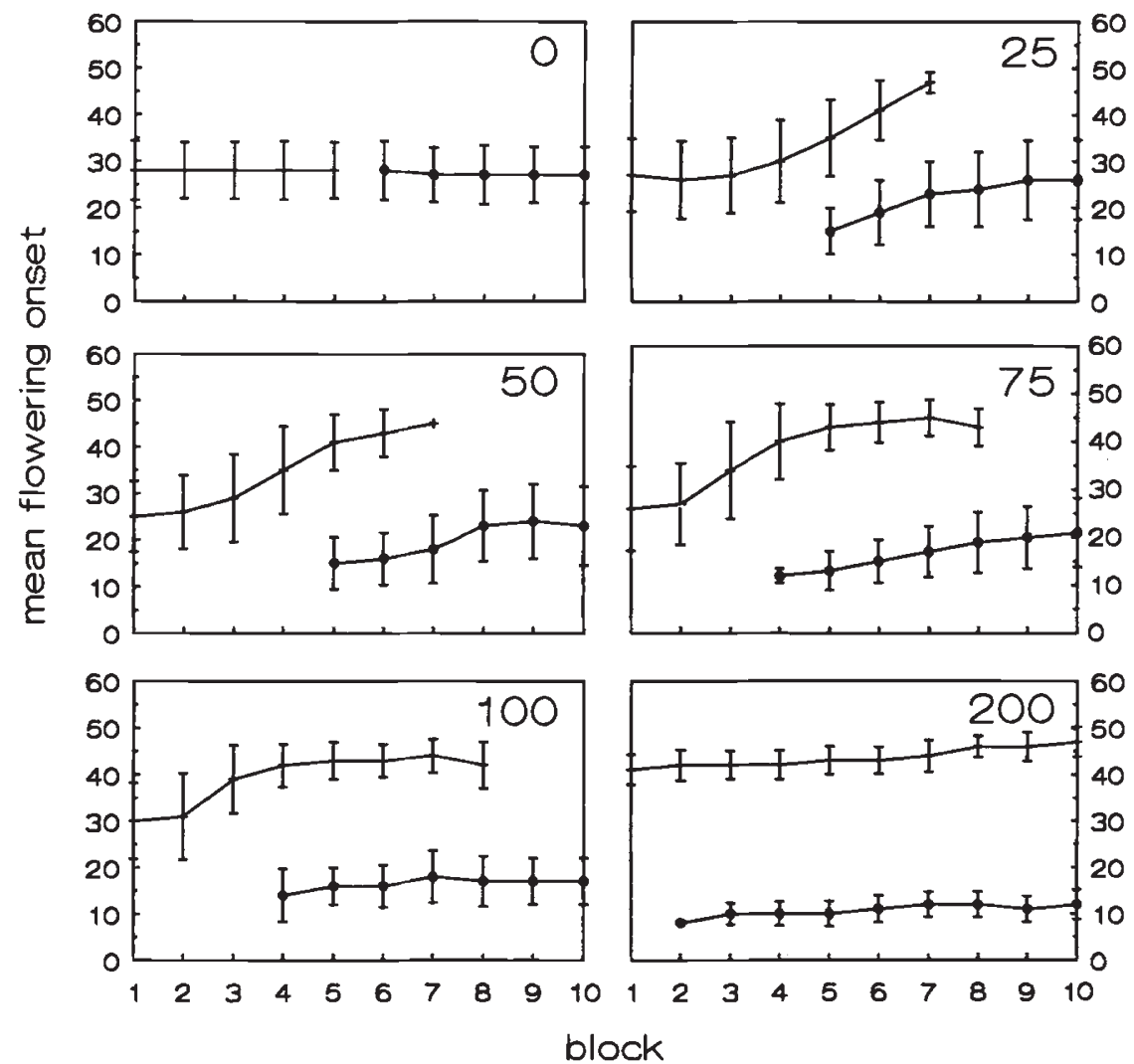



Fig. 11 Characteristics of the simulation run shown in Fig. 8. The divergence in flowering onset over generations (left axis); upper thick line: cytotype A; lower thick line cytotype $B$; given are population means \pm s.d. The percentage flowering time overlap (right axis) is indicated by a dotted line. The bar along the left axis indicates the flowering time duration (16 units).

placement differences between sympatric taxa are expected to be larger than between allopatric taxa. Displaced flowering times have been claimed in a number of wind-pollinated plant species (e.g. Agrostis tenuis and Anthoxanthum odoratum (McNeilly \& Antono- vics, 1968) and Pinus muricata (Millar, 1983). These would be cases of reinforcement rather than character displacement as post-zygotic isolation was not complete. However, alternative explanations may be equally likely (Stam, 1982; Butlin, 1989). Carter \& Murdy (1986) reported a likely case of displaced flowering times in diploid Talinum mengesii and its allotetraploid derivative $T$. teretifolium. Hybrids between these species are sterile. In the glasshouse, diurnal flowering time differences were shown to be larger between plants originating from sympatric populations than between plants from allopatric populations.

In many plant species cytotypes occur that produce unfit hybrids. It is remarkable that there are only few reports in the literature on sympatric populations of such cytotypes. Although this may be due to poor sampling or to ignorance, it may also be that sympatric populations are rare because reproductive character displacement has not been strong enough to allow stable co-existence. Alternatively, niche overlap and competitive exclusion may have prevented co-existence. More population biological studies of such situations are needed to elucidate this interesting problem. 


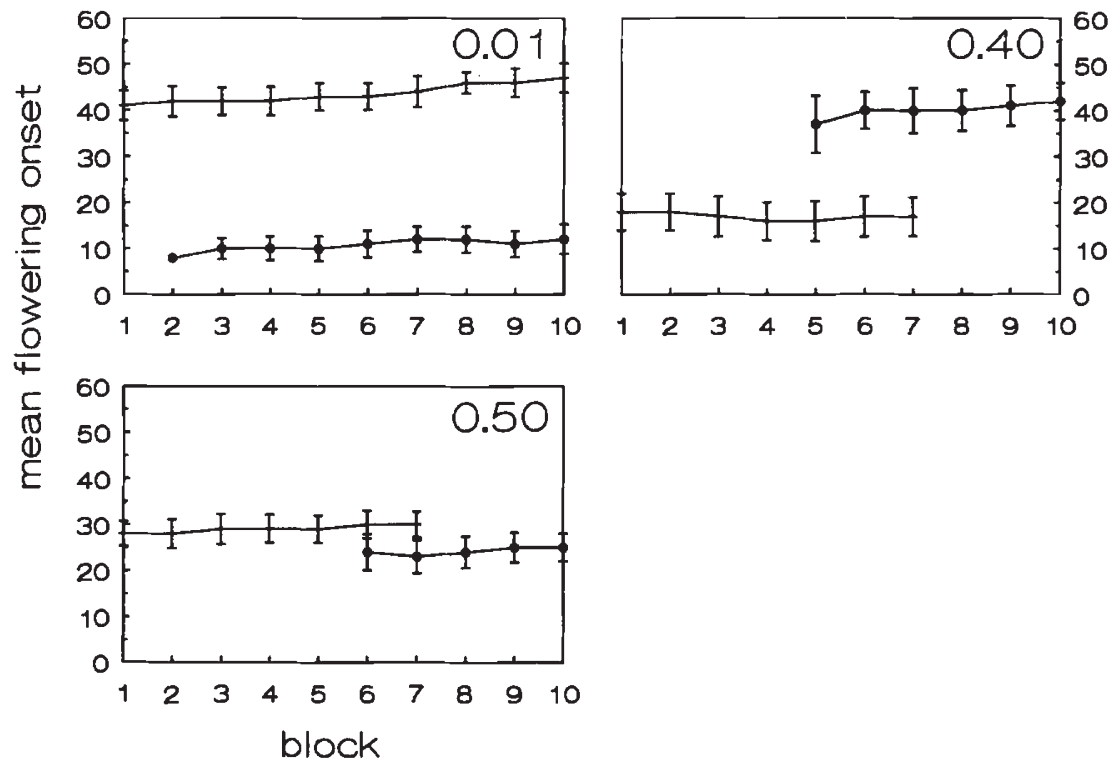

Fig. 12 Cross-sections of the flowering onset distributions after 200 generations for three different $\mathrm{O} / \mathrm{P}$ ratios: $0.01,0.40$ and 0.50 . Mean flowering onset per block \pm s.d. ( + : cytotype A; -: cytotype B).

\section{Acknowledgements}

We are grateful to Dr Piet Stam for his advice and to Drs Wilke van Delden and John Parker for their valuable comments on drafts of the paper. Nicole Tolmeyer and Marijke Hartog made most of the figures. This study was supported by the Foundation for Fundamental Biological Research (BION), which is subsidized by the Netherlands Organization for the Advancement of Scientific Research (NWO). This paper is Grassland Species Research Group Publication No. 183.

\section{References}

BARTON, N. H, AND HEWITT, G. M. 1985. Analysis of hybrid zones. Ann. Rev. Ecol. Syst., 16, 113-148.

BLAIR, W. F. 1955. Mating call and stage of speciation in the Microhyla olivacea $-M$. carolinensis complex. Evolution, 9, 469-480.

BOS, M., HARMENS, H. AND VRIELING, K. 1986. Gene flow in Plantago. 1. Gene flow and neighbourhood size in $P$. lanceolata. Heredity, 56, 43-54.

BUTLIN, R. 1987. Speciation by reinforcement. Trends Ecol. Evol., 2, 8-13.

BUTLIN, R. 1989. Reinforcement of premating isolation. In: Otte, D. and Endler, J. A. (eds) Speciation and its Consequences, pp. 85-110. Sinauer Associates, Sunderland, MA.

CAISSE, M. AND ANTONOvics, J. 1978. Evolution in closely adjacent plant populations. IX. Evolution of reproductive isolation in clinal populations. Heredity, 40, 371-384.

CARTER, M. E. B. AND MURDY, w. H. 1986. Divergence for sexual and asexual reproductive characters in Talinum mengesii (Portulacaceae). Bull. Torrey Bot. Club, 113, 259-267.
CROSBY, J. L. 1970. The evolution of genetic discontinuity computer models for the selection of barriers to interbreeding between subspecies. Heredity, 25, 253-297.

DOBZHANSKY, Th. 1951. Genetics and the Origin of Species. Columbia University Press, New York.

FELSENSTEIN, J. 1981. Skepticism towards Santa Rosalia, or why are there so few kinds of animals? Evolution, 35, 124-138.

FOX, G. A. AND KELLEY, C. K. 1993. Plant phenology: selection and neutrality. Trends Ecol. Evol., 8, 34-35.

FRASER, A. AND BURNELl, D. 1970. Computer Models in Genetics. McGraw Hill, New York.

GRANT, v. 1966. The selective origin of incompatibility barriers in the plant genus Gilia. Am. Nat., 100, 99-118.

JOHNSTON, S. A., DEN NIJS, T. P. M., PELOQUIN, S. J. AND HANNEMAN, R. E., Jr. 1980. The significance of genetic balance to endosperm development in interspecific crosses. Theor. Appl. Genet., 57, 5-9.

KAY, Q. O. N. 1969. The origin and distribution of diploid and tetraploid Tripleurospermum inodorum (L.) Schultz Bip. Watsonia, 7, 130-141.

KOOPMAN, K. F. 1950. Natural selection for reproductive isolation between Drosophila pseudoobscura and Drosophila persimilis. Evolution, 4, 135-148.

LEVIN, D. A. 1970. Reinforcement of reproductive isolation: plants vs. animals. Am. Nat., 104, 571-581.

LEVIN, D. A. 1975. Minority cytotype exclusion in local plant populations. Taxon, 24, 35-43.

LEVIN, D. A. AND ANDERSON, w. w. 1970. Competition for pollinators between simultaneously flowering species. $\mathrm{Am}$. Nat., 104, 455-467.

McNEILLY, T. AND ANTONOVICS, J. 1968. Evolution in closely adjacent plant populations. IV. Barriers to gene flow. Heredity, 23, 205-218.

MiLlaR, C. I. 1983. A steep cline in Pinus muricata. Evolution, 37, 311-319. 
MOORE, w. S. 1979. A single locus mass-action model of assortative mating, with comments on the process of speciation. Heredity, 42, 173-186.

MOORE, w. S. 1982. Assortative mating genes selected along a gradient. Heredity, 46, 191-195.

OLLERTON, J. AND LACK, A. J. 1992. Flowering phenology: an example of relaxation of natural selection? Trends Ecol. Evol., 7, 274-276.

PATERNIANI, E. 1969. Selection of reproductive isolation between two populations of maize, Zea mays L. Evolution, 23, 543-547.

RATHCKE, B. AND LACEY, E. P. 1985. Phenological patterns of terrestrial plants. Ann. Rev. Ecol. Syst., 16, 179-214.

ROHLF, F. J. AND SCHNELL, G. D. 1971. An investigation of the isolation by distance model. Am. Nat., 105, 295-324.

SANDERSON, N. 1989. Can gene flow prevent reinforcement? Evolution, 43, 1223-1235.

SOKAL, R. R. AND WARTENBERG, D. E. 1983. A test of spatial autocorrelation analysis using an isolation-by-distance model. Genetics, 105, 219-237.

SPENCER, H. G., MCARDLE, B. H. AND LAMBERT, D. M. 1986. A theoretical investigation of speciation by reinforcement. Am. Nat., 128, 241-262.
STAM, P. 1982. The evolution of reproductive isolation in closely adjacent plant populations through differential flowering time. Heredity, 50, 105-118.

TURNER, M. E., STEPHENS, J. C. AND ANDERSON, W. W. 1982. Homozygosity and patch structure in plant populations as a result of nearest-neighbor pollination. Proc. Natl. Acad. Sci. U.S.A., 79, 203-207.

VAN DIJK, P. 1991. Evolutionary Aspects of Polyploidy in Plantago media L. Ph.D. Thesis, University of Groningen.

VAN DIJK, P. AND VAN DELDEN, w. 1990. Evidence for autotetraploidy in Plantago media L. and comparisons between natural and artificial cytotypes concerning cell size and fertility. Heredity, 65, 349-357.

VAN DIJK, P., HARTOG, M. v. AND VAN DELDEN, w. 1992. Single cytotype areas in autopolyploid Plantago media L. Biol. $J$. Linn. Soc., 46, 315-331.

VAN DIJK, P., LAZARE, J. J., HARTOG, M. V. AND VAN DELDEN, w. 1994. On the coexistence of cytotypes of $P$. media L.: population structure, ecological differentiation and reproductive character displacement. J. Evol. Biol. (in press).

wOODELL, S. R. J. AND VALENTINE, D. H. 1961. Studies in British primulas. IX. Seed incompatibility in diploid-autotetraploid crosses. New Phytologist, 60, 282-294. 